\title{
Analysing the Behaviour of Students in Learning Management Systems with Respect to Learning Styles
}

\author{
Sabine Graf and Kinshuk \\ ${ }^{1}$ Vienna University of Technology, Women's Postgraduate College for \\ Internet Technologies, Vienna, Austria, sabine.graf@ieee.org \\ ${ }^{2}$ Athabasca University, School of Computing and Information Systems, \\ Athabasca, Canada, kinshuk@ieee.org
}

\begin{abstract}
Learning management systems (LMS) are successfully used in eeducation but they provide the same courses for all learners rather than considering the learners' individual needs. In recent years, more and more research is done on incorporating individual characteristics such as learning styles in technology enhanced learning. According to educational theories, learners with a strong preference for a specific learning style might have difficulties in learning if their learning style is not considered by the teaching environment. On the other hand, providing courses that fit to the individual learning styles makes learning easier for students. As a requirement for taking learning styles into consideration in LMS, the behaviour of students in online courses needs to be investigated. In this chapter, we analyse the behaviour of 43 students during an online course within an LMS with respect to their learning styles. The results show that learners with different preferences for learning styles act also differently in the course. From these results, information about the preferred way of learning and their favoured features in the LMS can be gained. On one hand, this information can be used to incorporate different features in a course in order to support different learning styles. On the other hand, the information can act as basis for providing adaptive courses. Moreover, we analysed the behaviour of students and their learning styles with respect to correlations. As a result, we found several significant correlations which can be used to investigate and develop an automatic approach for detecting learning styles based on the behaviour of learners in LMS.
\end{abstract}




\section{Introduction}

Learners have different ways of learning. Some learners prefer to work in groups, whereas others like to learn alone. Some prefer to learn concrete material such as data and facts; others prefer abstract content like theories and their underlying meaning. Besides, the students' perception of a course or a learning environment might be different. For some students, a course seems to be easy, whereas others have problems with learning in the same course. According to Jonassen and Grabowski [1], the reason can be seen in the individual differences of learners which also include their individual learning styles. Felder, for example, pointed out that learners with a strong preference for a specific learning style might have difficulties in learning if their learning style is not supported by the teaching environment $[2,3]$. On the other hand, incorporating learning styles makes learning easier and leads to better achievement. Bajraktarevic, Hall, and Fullick [4], for example, confirmed this by a study showing that students attending an online course that matches their preferred learning style (either sequential or global) achieved significantly better results than those who got delivered a course that did not match their learning style.

In web-based learning systems, more and more attention is paid on incorporating learning styles and providing courses that fit to the students' individual learning style. Some examples of such adaptive systems are CS383 [5], IDEAL [6], INSPIRE [7], and TANGOW [8].

While supporting adaptivity is a big advantage of these systems, they also have severe limitations. For example, adaptive systems lack integration, supporting only few functions of web-enhanced education, and the content of courses is not available for reuse [9]. On the other hand, learning management systems (LMS) such as Moodle [10], Blackboard [11], and WebCT [12] provide a lot of simple features to administer and create courses. As such, they have become very successful in e-education, but they provide very little or, in most cases, no adaptivity [13].

As a requirement for incorporating learning styles in LMS, analysis needs to be carried out about the behaviour of learners with respect to their learning styles. In this chapter, we investigate the behaviour of learners in an online course within Moodle [10]. Our investigations are based on the learning style model by Felder and Silverman [2], which is described in more detail in Section 2. Based on this model, we identified several patterns of behaviour (Section 3), which on the one hand seem to be relevant with respect to the learning style model and on the other hand are commonly used features in LMS. This concept makes our results applicable for other LMS as well. 
Analysing the Behaviour of Students in Learning Management Systems with Respect to Learning Styles 3

The performed study (Section 4) aims at two issues: Firstly, we investigate whether learners with different learning style preferences act differently in the online course. The results (Section 5) show the different preferences and needs of students with different learning styles. Since LMS currently provide the same course for each student, these results can act as the catalyst to make teachers and course developers aware of the needs of their students in order to incorporate these needs into the course development process by providing features for each learning style. Furthermore, the results can be used as recommendation for providing adaptivity based on learning styles in LMS.

Secondly, we investigate correlations between the learning style preferences and the behaviour of the students in the course. From these correlations, it is not only possible to draw conclusions from learning style preferences to the behaviour but also to obtain indications from the behaviour of students about their learning style preferences. These results provide information which can be used to investigate the identification of learning styles in LMS based on the actual behaviour of students during an online course.

The benefits of both analyses are discussed in detail in Section 6. In section 7 , we describe related work and point out differences to our work. Section 8 concludes the chapter.

\section{Felder-Silverman Learning Style Model}

While several learning style theories exist in the literature, e.g. the learning style models by Kolb [14] and Honey and Mumford [15], FelderSilverman learning style model (FSLSM) [2] seems to be the most appropriate for use in computer-based educational systems [5, 16]. Most other learning style models classify learners in few groups, whereas FSLSM describes the learning style of a learner in more detail, distinguishing between preferences on four dimensions.

The first dimension distinguishes between an active and a reflective way of processing information. Active learners learn best by working actively with the learning material, e.g. working in groups, discussing the material, or applying it. In contrast, reflective learners prefer to think about and reflect on the material.

The second dimension covers sensing versus intuitive learning. Learners with preference for a sensing learning style like to learn facts and concrete learning material. They tend to be more patient with details and more careful about their work. Furthermore, sensing learners tend to be more practi- 
cal than intuitive learners and like to relate the learned material to the real world. Intuitive learners prefer to learn abstract learning material, such as theories and their underlying meanings. They like to discover possibilities and relationships, and tend to be more innovative than sensing learners. This dimension differs from the active/reflective dimension in an important way: the sensing/intuitive dimension deals with the preferred source of information whereas the active/reflective dimension covers the process of transforming the perceived information into knowledge.

The third, visual/verbal dimension differentiates learners who remember best what they have seen, e.g. pictures, diagrams and flow-charts, and learners who get more out of textual representations, regardless of the fact whether they are written or spoken.

In the fourth dimension, the learners are characterized according to their understanding. Sequential learners learn in small incremental steps and therefore have a linear learning progress. They tend to follow logical stepwise paths in finding solutions. In contrast, global learners use a holistic thinking process and learn in large leaps. They tend to absorb learning material almost randomly without seeing connections, but after learning enough material they suddenly get the whole picture. Then they are able to solve complex problems and put things together in novel ways but find it difficult to explain how they did it. Since the whole picture is important for global learners, they tend to be more interested in overviews and a broad knowledge whereas sequential learners are more interested in details.

\section{Investigated Patterns of Behaviour}

The aim of our investigations is to analyse the behaviour of students in an online course with respect to their learning styles. These investigations are based on predefined patterns which on one hand are related to the FSLSM and on the other hand are based on commonly used features in LMS.

FSLSM is based on traditional learning rather than online learning and therefore describes the preferences of students in traditional learning environments. To apply FSLSM in online environments, some sort of mapping between the behaviour in traditional environments and in online environments is necessary. Therefore, we chose patterns in online environments that are related to the traditional behaviour and tested their significance with respect to learning styles.

Additionally, our findings should be applicable for LMS in general rather than only for Moodle. Since different LMS provide authors and course developers with the opportunity to integrate different features in an 
Analysing the Behaviour of Students in Learning Management Systems with Respect to Learning Styles 5

online course, we used only those features which are implemented in most LMS and which are also commonly used by authors and course developers.

The incorporated features include content objects, outlines, examples, self-assessment tests, exercises, and discussion forums. Furthermore, we investigated the navigation preferences of students in the course as well as the sequence in which they visited specific features. In the following paragraphs, a brief description of the features as well as the related patterns to each feature is provided.

Content objects are used to present the content of the course. These content objects can have different formats, depending on the LMS. For example, content can be presented as html-files or pdf-files. Related to the content objects, we considered the number of visits as well as the time learners spent on content objects. Additionally, we tracked the time learners spent on content objects including graphics.

We also included patterns regarding outlines of chapters since they are explicitly mentioned in FSLSM. Therefore, we again looked at the number of visits of outlines and the time learners spent on it.

Another feature is examples which aim at illustrating the theoretical content in a more concrete way. Again, the number of visits and the time learners spent on these objects are used as patterns.

Furthermore, self-assessment tests are included where students can check their acquired knowledge. Regarding these tests, we considered more detailed information such as the number of questions a learner answered, whether a learner performed all available tests at least once, the results a learner achieved, how often a learner revised his/her answers before submitting, how long a learner spent on the tests, and how long a learner checked his/her results. Furthermore, the questions contained in a test can be about facts or concepts, refer to an overview or to details, deal with interpreting or developing solutions, or can be based on graphics rather than on text. The results learners achieved on each kind of questions act as pattern as well.

Another element includes exercises which serve as practice area where students can try things out or answer questions about interpreting predefined solutions or developing new solutions. The number of visits and the time student spent on exercises is considered as pattern. Information about the number of revisions as well as students' performance on interpreting and developing solutions is gathered and combined with the data from selfassessment tests. 
For communication issues, discussion forum is considered. As patterns, we incorporated the number of visits to the forum, how long learners stayed at the forum, and how many messages they posted.

Additionally, we incorporated the navigation between learning objects as well as the number of logins in the course. We considered how often learning objects were skipped in the course sequence, how often learners jumped back to the previous learning object, as well as how often and how long they stayed at the course overview page.

Furthermore, we incorporated patterns regarding the sequence, in which learners visited specific features. We considered which feature was visited first and last in a course chapter, distinguishing between content objects, outlines, self-assessment tests, exercises, examples, and marked assignments. Moreover, we used the order in which content objects, selfassessment tests, exercises, and examples were visited by comparing, for instance, in how many course chapters a student was visiting a content object before or after he/she was performing a self-assessment test. This is done for all combinations of the four features, resulting in six patterns.

\section{Design of the Study}

In this section, information about the design of the study is provided. Therefore, the course itself and its structure are described. Subsequently, we introduce the instrument which was used for identifying learning styles according to FSLSM. Although Moodle provides already quite comprehensive tracking mechanisms, some extensions were necessary in order to track all information that we aimed at investigating in our study. The last subsection presents the performed extensions in Moodle.

\section{1 Description of the Course}

The study is based on the data from a laboratory course about Web Engineering which was taught at Vienna University of Technology, Austria, in summer term 2006. The course was divided into two parts, XML and Java. Only for the XML part, all features which were mentioned in the previous section such as content object, examples, exercises and so on, were included in Moodle [10]. Therefore, our investigations deal with the XML part of the course only.

The XML part itself consisted of three chapters that included 182 content objects (39 include graphics) and 14 examples in total. Students could solve 8 different exercises which allowed them to parse their entered 
Analysing the Behaviour of Students in Learning Management Systems with Respect to Learning Styles 7

source code and provided feedback. Self-assessment tests were provided for five topics, and included 123 questions overall.

Each chapter also included one marked assignment which had to be done in groups of two. Few days after the submission, each student had to present the solution individually and had to answer questions about it. At the end of the course, each student had to pass a written exam.

Although parts of the assignments were done in groups of two, the course was designed in a way that all students needed to learn everything and they were examined on all topics; hence the course was appropriate for investigation of individual learning.

\subsection{Instrument for Identifying Learning Styles}

In order to investigate the behaviour of students during the course with respect to their learning styles, these learning styles needed to be identified. Therefore, we used the Index of Learning Styles (ILS), a 44-item questionnaire developed by Felder and Soloman [3]. The ILS identifies learning styles according to FSLSM and is available online.

As mentioned earlier, each learner has a personal preference for each of the four dimensions of FSLSM. These preferences are expressed with values between +11 to -11 per dimension. This range comes from the 11 questions that are posed for each dimension. When answering a question, for instance, with an active preference, +1 is added to the value of the active/reflective dimension, whereas an answer for a reflective preference decreases the value by 1 .

The ILS is an often used and well investigated instrument to identify the learning style. An overview of studies dealing with analysing the response data of ILS as well as with verifying the reliability and validity of the instrument is provided by Felder and Spurlin [17].

\subsection{Extensions in Moodle for Tracking Students' Behaviour}

In order to make our results applicable for most LMS, we incorporated only commonly used features of LMS. On the other hand, the investigations about the behaviour regarding these features asked for detailed information. Although Moodle provides much information about learners' behaviour in the courses, the need for some extensions emerged. These extensions focused on two issues. On the one hand, they dealt with getting additional descriptions about the learning material in order to distinguish it and be able to draw conclusions about students' behaviour related to spe- 
cific kinds of learning material. On the other hand, extensions dealt with improving tracking mechanisms.

Moodle provides a number of different features to include in an online course. For our investigations and with respect to the above introduced patterns, only some of these features are of particular interest, namely the resources, the quiz, the assignments, and the forum. In Moodle, learning material regarding all proposed features can be created by using these four different types of learning objects. But for investigations regarding the learners' behaviour, the learning material has to be distinguished with respect to the proposed features and for some features, an additional description of the material is necessary. In most cases, this differentiation and additional descriptions are not supported by Moodle. Therefore, we added the possibility for authors to specify information about the created learning material by the use of meta-data. For resources, we ask authors to specify whether the learning objects can be considered as content objects, outlines, or examples. Furthermore, we provide authors the opportunity to specify whether the material includes graphics. For quizzes, we give the possibility to specify whether the quiz can be considered as exercise or as selfassessment test. Moreover, the authors are asked to specify the questions in more detail by indicating whether the questions are about facts or concepts, refer to an overview or to details, are based on graphics rather than on text, or deal with interpreting predefined solutions or developing new solutions. This detailed specification provides information about which kind of question a learner handles easily or with difficulty.

Since Moodle provides comprehensive tracking mechanisms, only one extension was necessary. This extension deals with the tracking of behaviour during students are performing quizzes. So far, only the final answers of the questions were stored. In order to get more detailed information about what learners are doing during a quiz, we extended the tracking function by storing each answer which is given by a learner, even if this answer is revised later. When the question asks for a textual answer rather than for choosing between predefined options, additionally the sequence of keys is tracked with attention to the delete and backspace key. This additional data provides information about how often students are revising their answers.

\section{Results}

We investigated two different issues within this study: Firstly, we analysed the given data in order to draw conclusions about whether students with 
Analysing the Behaviour of Students in Learning Management Systems with Respect to Learning Styles 9

different learning styles, or more precisely with different preferences for the questions of ILS, act differently in the online course. Secondly, we aimed at finding correlations between the answers to the questions and the behaviour of students during the course.

43 students participated in our study. Since all students have either a visual or a balanced learning style and no student indicated a verbal style, further investigations are focused only on the active/reflective, sensing/intuitive, and sequential/global dimension.

\subsection{Behaviour vs. Learning Style Preferences}

In order to identify significant differences of behaviour in the online course from different answers to questions of the ILS, we divided the students for each question, according to their answer (+1 or -1$)$, into two groups. Then we tested these two groups respectively for significant difference for each pattern of behaviour described in Section 3.

Two tailed t-test was applied for patterns where data was normal distributed and two tailed Mann-Whitney U test (u-test) for patterns where data was not normal distributed. To check whether data was normal distributed, we used Kolmogorov-Smirnov test.

The results are presented in Table 1 . Only significant values $(\mathrm{p}<0.05)$ are shown. The table shows the patterns and respectively the ILS questions which lead to a significant result according to t-test or $\mathrm{u}$-test. The $\mathrm{T}$ and $\mathrm{U}$ values as well as whether t-test or u-test was conducted, the significance level (p), and the direction of the relationship (d) is presented. Regarding the direction, 1 indicates that a high value concerning the pattern refers to the group answered the ILS question with +1 and vice versa.

Regarding the patterns dealing with visiting specific features first or last in a course chapter, we considered only patterns where the occurrence was higher than 5. Therefore, only assignments and examples were considered with respect to the first visited learning object, and content objects, examples, self-assessments, exercises, and assignments were considered regarding the last visited learning object.

In the following discussion, for all significant results the respective question is in semantic relation with the pattern unless mentioned otherwise.

\subsubsection{Active/Reflective Dimension}

According to the results of the active/reflective dimension, it can be seen that spending more time on examples and dealing more intensively with 
outlines (visiting and spending time) seems to be significant for reflective learning. These findings are in agreement with FSLSM, since reflective learners are described as learners who think and reflect more deeply about the provided learning material.

Table 1. Results of t-test/u-test

\begin{tabular}{|c|c|c|c|c|c|c|}
\hline & Pattern & Question & $\begin{array}{l}\text { t-test/ } \\
\text { u-test }\end{array}$ & $\mathrm{T}$ or $\mathrm{U}$ & $\mathrm{p}$ & $\mathrm{d}$ \\
\hline \multirow{14}{*}{ 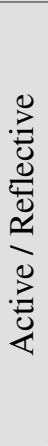 } & outline_visit & $\mathrm{q} 29$ & $\mathrm{t}$ & -2.24 & 0.031 & -1 \\
\hline & outline_stay & q29 & $\mathrm{u}$ & 65.50 & 0.002 & -1 \\
\hline & example_stay & $\mathrm{q} 33$ & $\mathrm{u}$ & 143.50 & 0.045 & -1 \\
\hline & selfass_stay_results & q5 & $\mathrm{u}$ & 25 & 0.007 & -1 \\
\hline & ques_visit & q5 & $\mathrm{u}$ & 154.00 & 0.050 & 1 \\
\hline & ques_facts & q5 & $\mathrm{t}$ & 3.21 & 0.005 & 1 \\
\hline & ques_interpret & q9 & $\mathrm{t}$ & -3.32 & 0.004 & -1 \\
\hline & forum_visit & $\mathrm{q} 25$ & $\mathrm{t}$ & -2.92 & 0.006 & -1 \\
\hline & navigation_overview_stay & q13 & $\mathrm{t}$ & 2.17 & 0.036 & 1 \\
\hline & navigation_overview_stay & $\mathrm{q} 25$ & $\mathrm{t}$ & -3.02 & 0.005 & -1 \\
\hline & sequence_exercise/example & $\mathrm{q} 21$ & $\mathrm{u}$ & 136.50 & 0.049 & 1 \\
\hline & sequence_selfass_last & $\mathrm{q} 25$ & $\mathrm{u}$ & 177.50 & 0.043 & -1 \\
\hline & sequence_selfass_last & q29 & $\mathrm{u}$ & 129.50 & 0.044 & -1 \\
\hline & sequence_assignment_last & q13 & $\mathrm{u}$ & 164.50 & 0.040 & 1 \\
\hline \multirow{17}{*}{ 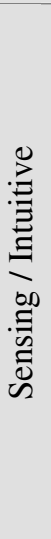 } & content_visit & q26 & $\mathrm{t}$ & 2.69 & 0.012 & 1 \\
\hline & outline_visit & $\mathrm{q} 22$ & $\mathrm{t}$ & 2.04 & 0.048 & 1 \\
\hline & outline_stay & q34 & $\mathrm{u}$ & 123.00 & 0.036 & -1 \\
\hline & example_visit & $\mathrm{q} 2$ & $\mathrm{u}$ & 104.00 & 0.044 & 1 \\
\hline & example_stay & q10 & $\mathrm{u}$ & 111.50 & 0.043 & 1 \\
\hline & ques_overview & $\mathrm{q} 42$ & $\mathrm{t}$ & -2.61 & 0.018 & -1 \\
\hline & ques_revisions & q10 & $\mathrm{t}$ & 2.47 & 0.021 & 1 \\
\hline & forum_stay & $\mathrm{q} 10$ & $\mathrm{t}$ & 2.79 & 0.008 & 1 \\
\hline & forum_stay & $\mathrm{q} 22$ & $\mathrm{t}$ & 2.63 & 0.012 & 1 \\
\hline & forum_post & $\mathrm{q} 22$ & $\mathrm{u}$ & 117.00 & 0.001 & 1 \\
\hline & navigation_back & $\mathrm{q} 22$ & $\mathrm{u}$ & 161.50 & 0.048 & 1 \\
\hline & sequence_content/selfass & q10 & $\mathrm{u}$ & 104.00 & 0.011 & 1 \\
\hline & sequence_exercise/selfass & $\mathrm{q} 42$ & $\mathrm{u}$ & 153.00 & 0.029 & -1 \\
\hline & sequence_example_first & q26 & $\mathrm{u}$ & 154.00 & 0.003 & 1 \\
\hline & sequence_assignment_first & q10 & $\mathrm{u}$ & 99.50 & 0.014 & 1 \\
\hline & sequence_example_last & q10 & $\mathrm{u}$ & 135.00 & 0.022 & 1 \\
\hline & sequence_example_last & $\mathrm{q} 38$ & $\mathrm{u}$ & 170.00 & 0.035 & 1 \\
\hline \multirow{15}{*}{ 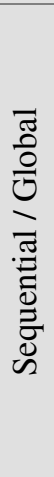 } & outline_visit & q12 & $\mathrm{t}$ & 2.99 & 0.005 & $\overline{1}$ \\
\hline & outline_stay & $\mathrm{q} 44$ & $\mathrm{u}$ & 114.50 & 0.005 & 1 \\
\hline & selfass_visit_different & $\mathrm{q} 36$ & $\mathrm{u}$ & 101.00 & 0.028 & 1 \\
\hline & selfass_stay_results & q20 & $\mathrm{u}$ & 33.00 & 0.024 & 1 \\
\hline & ques_concepts & $\mathrm{q} 44$ & $\mathrm{t}$ & -2.11 & 0.049 & -1 \\
\hline & ques_graphics & $\mathrm{q} 32$ & $\mathrm{t}$ & 2.86 & 0.010 & 1 \\
\hline & ques_revisions & $\mathrm{q} 28$ & $\mathrm{t}$ & 3.04 & 0.007 & 1 \\
\hline & forum_post & q20 & $\mathrm{u}$ & 149.00 & 0.014 & 1 \\
\hline & navigation_skip & $\mathrm{q} 20$ & $\mathrm{u}$ & 176.00 & 0.038 & -1 \\
\hline & navigation_overview_visit & $\mathrm{q} 44$ & $\mathrm{t}$ & -2.71 & 0.010 & -1 \\
\hline & sequence_content/exercise & $\mathrm{q} 28$ & $\mathrm{u}$ & 34.00 & 0.020 & 1 \\
\hline & sequence_content/example & $\mathrm{q} 4$ & $\mathrm{u}$ & 76.50 & 0.038 & -1 \\
\hline & sequence_content_last & q12 & $\mathrm{u}$ & 171.00 & 0.021 & 1 \\
\hline & sequence_assignment_last & $\mathrm{q} 24$ & $\mathrm{u}$ & 145.50 & 0.037 & -1 \\
\hline & sequence_assignment_last & q32 & $\mathrm{u}$ & 127.00 & 0.007 & -1 \\
\hline
\end{tabular}


Analysing the Behaviour of Students in Learning Management Systems with Respect to Learning Styles 11

When looking at the sequence of visited learning objects, we found a significant preference for reflective learners to visit examples first and perform then exercises. In contrast, active learners preferred to perform exercises first and looked then at examples. According to FSLSM, active learners prefer to try things out and work actively, as they can do with exercises. By looking at examples, active learners see how others have solved a problem rather than being able to solve the problem by themselves. Therefore, they preferred exercises over examples and focused on exercises first. On the other hand, reflective learners gain more from examples, where they can reflect on an already given solution. Therefore, they looked at examples first and afterwards performed exercises.

Furthermore, it can be seen that reflective learners performed better on questions about interpreting predefined solutions (in terms of source code). This is again in line with the argumentation above. Moreover, they spent more time on looking at the results of their self-assessment tests. Again, this behaviour can be referred to the preference of reflecting.

In addition, results also show that reflective learners visited the forum significantly more often than active learners. This is because the forum in the course was mainly used for asking and clarifying questions regarding the assignments which were then answered by a tutor or a teacher. When the forum would be used for active discussions between students, maybe active learners would visit the forum more often.

Regarding active learning, it can be also seen that learners with an active preference performed significantly more self-assessment questions than reflective learners. This is in agreement with FSLSM as well, since active learners are characterized to prefer trying things out. It seems also to be significant that active learners performed better on questions dealing with facts. Further investigations about this finding need to be done since FSLSM does not include this behaviour in their description of an active/reflective learning style.

Considering the preferred first and last learning objects of active and reflective learners in a course chapter, it can be seen that reflective learners seem to perform self-assessment tests more often as last object in a course chapter than active learners. In contrast, active learners tended more often to submit their assignment and then went to the next course chapter. Since we also found out that active learners performed self-assessment test more often, these findings indicate that active learners used self-assessment tests as support for doing their assignments. In contrast, reflective learners were using these self-assessment tests also after the submission for preparing themselves for the presentation or the exam. 
When looking at the pattern indicating how long students spent on the overview page, it can be seen that for one question, students answering with an active preference spent more time on it and for another question students with a reflective preference did. Hence, it seems that a preference for an active or reflective learning style does not provide significant information about this pattern.

\subsubsection{Sensing/Intuitive Dimension}

Sensing learners are described by Felder and Silverman as learners who prefer concrete material. This can be also seen by our findings, showing that sensing learners visited more often examples and spent more time there than intuitive learners. Moreover, the results show that sensing learners started a course chapter more often with an example than intuitive learners. Also, their last visited learning object in a course chapter was more often an example than for intuitive learners. This indicates that sensing learners were using examples for their preparation for the presentation of the assignments and the written exam.

Another characteristic of sensing learners according to FSLSM is that they tend to be more patient with details and careful about their work. Looking at the pattern about revising their answers in self-assessment tests and exercises, it can be seen that learners with a sensing preference significantly more often changed their answers. It can also be seen that sensing learners spent more time in the forum and posted more often than intuitive learners. So, it can be argued that due to their preference for details, they wanted to clarify the specifications by asking in forums and were also interested in the questions and answers of others. Again, when the forum would be used more for discussion, these results may change. As can be seen from the results, sensing learners also tended to visit content objects and outlines more often and also navigated back more often to the previous page. This behaviour may also results from their patience and accuracy.

Another characteristic of sensing learners is that they tend to be more practical oriented and more interested in the application of the learned material in the real world. According to our results, it can be seen that sensing learners tended to start a course chapter more often with looking at the assignment than intuitive learners did. This behaviour may be due to their interest in applications. On the one hand, the assignments present the tasks which have to be done for the course chapter, but on the other hand, assignments are programming tasks that also show how the learned material can be applied.

Intuitive learners are characterized by Felder and Silverman as learners who like challenges. From our results, this is indicated by the sequence of 
Analysing the Behaviour of Students in Learning Management Systems with Respect to Learning Styles 13

visited learning objects. It can be seen that intuitive learners had a higher preference than sensing learners for performing self-assessment tests first and afterwards looking at the content objects. Also, we found from the results, that intuitive learners tried to do exercises first and then performed self-assessment tests. In our setting, exercises can be considered as more challenging since they ask students for programming tasks, whereas selfassessment tests provide students with the opportunity to check their theoretical knowledge about the learning material and are less comprehensive.

Two more significant patterns could be found for intuitive learners. One is dealing with the time students spent on outlines, the other one is about the results achieved for questions about overview. The second one may be explained by the preference of details for sensing learners and that they therefore achieve worse than intuitive learners on questions about overview. However, further investigations are necessary for both relations with regard to FSLSM.

\subsubsection{Sequential/Global Dimension}

According to FSLSM, a main characteristic of sequential learners is that they learn in a linear way, going through the material step by step. Accordingly, our results show that learners with a sequential preference tended to cover all/more topics of self-assessment tests and that they dealt more often with outlines which indicates that they started at the beginning of each chapter rather than jumping in and starting somewhere in between. Moreover, we found that sequential learners significantly more often visited the content first and afterwards performed exercises, as it was recommended in the course structure. In contrast, global learners tend to prefer a more non-sequential path through the course material. This can be seen when looking at the results of skipping learning objects which show that global learners skipped learning objects more often.

From our results, it can also be seen that learners with a global preference visited more often the course overview page. This is in agreement with FSLSM, since global learners are described to prefer getting an overview of the topic/course. While for global learners the overview is very important, sequential learners are more inclined to the details. According to Felder and Spurlin [17], it has been proven that the sequential/global dimension correlates slightly with the sensing/intuitive dimension. This may be caused due to the overlapping of the preference for details. Accordingly, our results show that sequential learners posted more often in the forum, looked more detailed at the results of their tests, and made more revisions when answering questions. In contrast, global learners performed 
significantly better on questions about concepts than sequential learners. Moreover, it was found that global learners had higher preference for submitting the assignments and then going to the next course chapter, whereas for sequential learners we found a preference for content objects as the last visited material in a course chapter. Again, this gives indication that sequential learners tend to be more accurate and careful by preparing themselves for the presentations and the exam after submitting the assignments.

Sequential learners seem to perform also better on questions about graphics. This might be because they remember better the details of the graphics. However, further investigations on this issue needs to be done.

Another result that needs further investigations is dealing with the preferred sequence of visiting examples and content. According to our results, sequential learners visited more often examples before content objects which is not in agreement with the recommended order of the course structure but might be explained by the correlation to the sensing learning style, where examples play an important role to facilitate learning for sensing learners.

\subsection{Correlations between Behaviour and Learning Style Preferences}

The previous analysis pointed out relations where learners who answered questions of ILS differently also acted differently in the online course. In the next analysis, we investigate the correlation between both, answers of ILS questions and the behaviour of the learners in the course based on the specified patterns. Thus, the resulting relations additionally allow drawing conclusions from the behaviour of the learners to their preferences of learning styles.

Since the values of the patterns are on a continuous scale and the possible answers to the questions of ILS can only be either +1 or -1 , pointbiserial correlation was performed using SPSS. Table 2 presents the results of the point-biserial correlation analysis. Again, only significant results are shown $(\mathrm{p}<0.05)$. Furthermore, we included only patterns where more than 5 students had a value greater than zero, as done in the previous analysis.

From the results, it can be seen that most of the significant relations found by t-test and u-test were also found by the point-biserial correlation. Therefore, in the following subsections, we discuss only the additional relations as well as relations which were found by t-test or u-test but were not confirmed by correlation analysis. 
Analysing the Behaviour of Students in Learning Management Systems with Respect to Learning Styles 15

Table 2. Results of correlation analysis

\begin{tabular}{|c|c|c|c|c|c|}
\hline & Pattern & Question & $\mathrm{rpb}$ & $\mathrm{p}$ & $\mathrm{d}$ \\
\hline \multirow{16}{*}{ 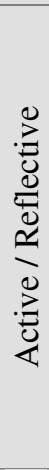 } & content_stay_graphics & $q 21$ & 0.34 & 0.037 & 1 \\
\hline & outline_visit & q29 & -0.33 & 0.031 & -1 \\
\hline & outline_stay & $\mathrm{q} 21$ & -0.34 & 0.026 & -1 \\
\hline & outline_stay & q29 & -0.43 & 0.004 & -1 \\
\hline & example_visit & $\mathrm{q} 33$ & -0.31 & 0.042 & -1 \\
\hline & selfass_visit_different & q5 & 0.35 & 0.022 & 1 \\
\hline & selfass_stay_results & $\mathrm{q} 1$ & -0.49 & 0.016 & -1 \\
\hline & ques_visit & q5 & 0.43 & 0.004 & 1 \\
\hline & ques_facts & q5 & 0.59 & 0.005 & 1 \\
\hline & ques_interpret & q9 & -0.64 & 0.004 & -1 \\
\hline & ques_develop & q5 & -0.64 & 0.036 & -1 \\
\hline & forum_visit & $\mathrm{q} 25$ & -0.41 & 0.006 & -1 \\
\hline & navigation_overview_stay & $\mathrm{q} 13$ & 0.32 & 0.036 & 1 \\
\hline & navigation_overview_stay & $\mathrm{q} 25$ & -0.43 & 0.004 & -1 \\
\hline & sequence_exercise/example & $\mathrm{q} 21$ & 0.34 & 0.025 & 1 \\
\hline & sequence assignment last & q13 & 0.33 & 0.030 & 1 \\
\hline \multirow{21}{*}{ } & content_visit & q26 & 0.39 & 0.009 & 1 \\
\hline & outline_visit & $\mathrm{q} 22$ & 0.30 & 0.048 & 1 \\
\hline & example_stay & $\mathrm{q} 10$ & 0.35 & 0.023 & 1 \\
\hline & example_stay & $\mathrm{q} 42$ & -0.43 & 0.004 & -1 \\
\hline & ques_detail & $\mathrm{q} 10$ & 0.43 & 0.050 & 1 \\
\hline & ques_overview & $\mathrm{q} 42$ & -0.52 & 0.018 & -1 \\
\hline & ques_develop & $\mathrm{q} 34$ & 0.66 & 0.028 & 1 \\
\hline & ques_revisions & $\mathrm{q} 10$ & 0.46 & 0.021 & 1 \\
\hline & exercise_visit & $\mathrm{q} 10$ & 0.38 & 0.011 & 1 \\
\hline & exercise_stay & $\mathrm{q} 10$ & 0.39 & 0.010 & 1 \\
\hline & forum_stay & $\mathrm{q} 10$ & 0.40 & 0.008 & 1 \\
\hline & forum_stay & $\mathrm{q} 22$ & 0.38 & 0.012 & 1 \\
\hline & forum_post & $\mathrm{q} 22$ & 0.48 & 0.001 & 1 \\
\hline & sequence_content/selfass & $\mathrm{q} 10$ & 0.43 & 0.004 & 1 \\
\hline & sequence_content/selfass & $\mathrm{q} 22$ & 0.33 & 0.032 & 1 \\
\hline & sequence_exercies/selfass & $\mathrm{q} 42$ & -0.32 & 0.038 & -1 \\
\hline & sequence_example_first & $\mathrm{q} 26$ & 0.45 & 0.002 & 1 \\
\hline & sequence_assignment_first & $\mathrm{q} 10$ & 0.38 & 0.013 & 1 \\
\hline & sequence_exercise_last & $\mathrm{q} 10$ & 0.35 & 0.021 & 1 \\
\hline & sequence_example_last & $\mathrm{q} 10$ & 0.37 & 0.015 & 1 \\
\hline & sequence_example_last & $\mathrm{q} 38$ & 0.31 & 0.045 & 1 \\
\hline \multirow{22}{*}{ 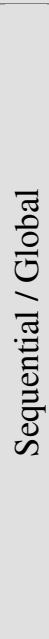 } & outline_visit & $\mathrm{q} 12$ & 0.42 & 0.005 & 1 \\
\hline & outline_stay & q44 & 0.34 & 0.024 & 1 \\
\hline & selfass_stay & q12 & -0.41 & 0.038 & -1 \\
\hline & selfass_stay & q16 & -0.40 & 0.042 & -1 \\
\hline & selfass_stay & $\mathrm{q} 20$ & -0.39 & 0.046 & -1 \\
\hline & selfass_visit_different & $\mathrm{q} 36$ & 0.34 & 0.024 & 1 \\
\hline & selfass_stay_results & $\mathrm{q} 28$ & 0.52 & 0.010 & 1 \\
\hline & ques_concepts & $\mathrm{q} 44$ & -0.45 & 0.049 & -1 \\
\hline & ques_graphics & $\mathrm{q} 32$ & 0.56 & 0.010 & 1 \\
\hline & ques_develop & $\mathrm{q} 20$ & -0.78 & 0.004 & -1 \\
\hline & exercise_stay & $\mathrm{q} 40$ & 0.33 & 0.032 & 1 \\
\hline & forum_post & $\mathrm{q} 20$ & 0.35 & 0.021 & 1 \\
\hline & forum_post & $\mathrm{q} 32$ & -0.33 & 0.031 & -1 \\
\hline & navigation_skip & $\mathrm{q} 40$ & 0.33 & 0.032 & 1 \\
\hline & navigation_overview_visit & $\mathrm{q} 44$ & -0.39 & 0.010 & -1 \\
\hline & sequence_content/selfass & q28 & 0.31 & 0.045 & 1 \\
\hline & sequence_content/exercise & $\mathrm{q} 28$ & 0.39 & 0.010 & 1 \\
\hline & sequence_exercise_last & $\mathrm{q} 12$ & 0.30 & 0.047 & 1 \\
\hline & sequence_exercise_last & $\mathrm{q} 28$ & 0.41 & 0.007 & 1 \\
\hline & sequence_content_last & $\mathrm{q} 12$ & 0.34 & 0.028 & 1 \\
\hline & sequence_assignment_last & $\mathrm{q} 24$ & -0.33 & 0.033 & -1 \\
\hline & sequence_assignment_last & $\mathrm{q} 32$ & -0.38 & 0.013 & -1 \\
\hline
\end{tabular}




\subsubsection{Active/Reflective Dimension}

Regarding the active/reflective dimension, additionally a relation can be seen between active learners and their preference for performing most or all self-assessment tests. This result is in agreement with FSLSM. The preference of reflective learners to finish a chapter with a self-assessment test more often than active learners could not be confirmed according to correlation analysis.

Furthermore, a relation can be seen between active learners and their interest in graphics. This interest in graphics may be explained by the fact that active learners tend to be less interested in reading and reflecting about text but instead look more details at graphics. Nevertheless, further investigations seem to be necessary since this behaviour is not explicitly described according to FSLSM.

While the time spent on examples could not be confirmed as an indication for a reflective preference, the number of visits was found as significant pattern. Regarding the performance on questions dealing with interpretation and development of source code, both seem to correlate with a reflective preference according to the results of the correlation analysis.

\subsubsection{Sensing/Intuitive Dimension}

While for learners with a sensing preference the number of visits of examples seems to be not significant according to the calculated correlation, exercises plays an important role. The number as well as the time spent on exercises correlates significantly with a sensing learning preference. Furthermore, it can be seen that learners with a sensing preference performed more often exercises as their last learning object in a chapter. This indicates that they used exercises to prepare themselves for the oral presentations and the written exam. The preferences of sensing learners for exercises are in agreement with FSLSM.

Regarding the time spent on examples, a significant correlation is found for a sensing as well as for an intuitive learning preference which necessitate further investigations.

An additional relation between a sensing learning preference and a better performance in questions about details and code development was found. Both are in agreement with FSLSM.

The impact of navigating to previous learning objects could not be confirmed by the results of the correlation analysis. Also the pattern indicating that intuitive learners spent more time on outlines was not found as significant according to correlation analysis. Anyway, this pattern is not explicitly supported by FSLSM. 
Analysing the Behaviour of Students in Learning Management Systems with Respect to Learning Styles 17

\subsubsection{Sequential/Global Dimension}

Regarding the sequential/global dimension, results show that a correlation was found indicating that learners with a global preference spent more time on self-assessment tests and performed better when developing source code. This is in line with FSLSM since the self-assessment tests are based on the learning material and therefore can be answered more easily when learning the material step by step, which tends to be the preferred way of learning for sequential learners. In contrast, for developing source code, more overview knowledge about the concepts is necessary, which tend to favour global learners.

According to the results of the correlation analysis, two further patterns were found, indicating the step-by-step navigation of sequential learners. First, it can be seen that sequential learners more often visited content objects before they performed self-assessment tests, as it is recommended by the course structure. In contrast to the u-test in the previous section, we did not found a significant correlation indicating that sequential learners looked at examples before they went to the content objects. Anyway, this pattern needs further analysis since it is not explicitly supported by FSLSM.

According to the u-test, sequential learners tended to look more often at content objects before they went to the next chapter. Regarding correlation analysis, an additional preference for exercises as last learning object of a chapter was found. However, both patterns indicate that sequential learners tend to be more accurate and prepare themselves for the presentation and the exam. On the other hand, the preference for assignments as last learning object of a chapter for global learners was confirmed by the correlation analysis.

Another correlation was found between the time students spent on exercises and a sequential learning preference. This relation needs further investigations with respect to FSLSM. Regarding the number of postings, once a positive and once a negative correlation was found. A similar disagreement was found for skipping learning material since u-test and correlation analysis indicate different directions of the relationship. Therefore, further investigations are necessary for both of these cases. Furthermore, the relation for revising answers in self-assessment tests and exercises could not be confirmed by correlation analysis. 


\section{Benefits}

Table 3 summarizes the results of our study. These results show that learners with different preferences for learning styles act differently in the online course. They used different features such as examples, exercises, and so on with different frequency, performed differently on specific kinds of questions, navigated differently through the course, and visited particular features in a different sequence. The results can also be interpreted in a way that each feature is needed to support a specific learning style and therefore plays an important role in the course. According to Felder and Silverman [2], learners might have difficulties in learning if their learning style is not supported by the teaching environment. As a solution, they recommended to provide courses with many different features which support different learning styles rather than providing courses that suit only one learning style.

The results of this study can act as catalyst to make teachers and course developers aware of the different needs of their students and also the different ways of learning from the course material. The results point out the preferences of learners with different learning styles. Furthermore, it can be seen that all investigated features are used differently at least regarding one learning style dimension. This shows the importance of each feature and highlights the requirement for providing different features to support each learning style.

At the current stage, learning management systems provide the same course for each learner. Learners then have the possibility to use the provided learning material in different ways and as can be seen from our results, they also do so. Besides providing a high amount of learning material that includes some features for each learning style, courses can also be adapted to the individual learning styles of learners. For providing proper adaptivity, it is important to know the preferences of learners with respect to their learning styles. Since FSLSM is developed for learning in traditional learning environments rather than for learning in technology enhanced learning environments, the behaviour of students in such environments has to be investigated and incorporated in the design of adaptation features. Accordingly, the results of this study can be used as basis for the generation process of adaptation features, especially in learning management systems. 
Analysing the Behaviour of Students in Learning Management Systems with Respect to Learning Styles 19

Table 3. Summary of the results

\begin{tabular}{|c|c|c|c|c|c|c|}
\hline \multirow[b]{2}{*}{ Patterns } & \multicolumn{2}{|c|}{ Active / Reflective } & \multicolumn{2}{|c|}{ Sensing / Intuitive } & \multicolumn{2}{|c|}{ Sequential / Global } \\
\hline & t/u-test & corr. & t/u-test & corr. & t/u-test & corr. \\
\hline $\begin{array}{l}\text { content_visit } \\
\text { content_stay_graphics }\end{array}$ & & $\checkmark$ & $\checkmark$ & $\checkmark$ & & \\
\hline outline_visit & $\checkmark$ & $\checkmark$ & $\checkmark$ & $\checkmark$ & $\checkmark$ & $\checkmark$ \\
\hline outline_stay & $\checkmark$ & $\checkmark$ & $\checkmark$ & & $\checkmark$ & $\checkmark$ \\
\hline example_visit & & $\checkmark$ & $\checkmark$ & & & \\
\hline example_stay & $\checkmark$ & & $\checkmark$ & & & \\
\hline exercise_visit & & & & $\checkmark$ & & \\
\hline exercise_stay & & & & $\checkmark$ & & $\checkmark$ \\
\hline selfass_stay & & & & & & $\checkmark$ \\
\hline selfass_visit_different & & $\checkmark$ & & & $\checkmark$ & $\checkmark$ \\
\hline selfass_stay_results & $\checkmark$ & $\checkmark$ & & & $\checkmark$ & $\checkmark$ \\
\hline ques_visit & $\checkmark$ & $\checkmark$ & & & & \\
\hline ques_revisions & & & $\checkmark$ & $\checkmark$ & $\checkmark$ & \\
\hline ques_facts & $\checkmark$ & $\checkmark$ & & & & \\
\hline ques_concepts & & & & & $\checkmark$ & $\checkmark$ \\
\hline ques_detail & & & & $\checkmark$ & & \\
\hline ques_overview & & & $\checkmark$ & $\checkmark$ & & \\
\hline ques_graphics & & & & & $\checkmark$ & $\checkmark$ \\
\hline ques_develop & & $\checkmark$ & & $\checkmark$ & & $\checkmark$ \\
\hline ques_interpret & $\checkmark$ & $\checkmark$ & & & & \\
\hline forum_visit & $\checkmark$ & $\checkmark$ & & & & \\
\hline forum_stay & & & $\checkmark$ & $\checkmark$ & & \\
\hline forum_post & & & $\checkmark$ & $\checkmark$ & $\checkmark$ & \\
\hline navigation_skip & & & & & $\checkmark$ & $\checkmark$ \\
\hline navigation_back & & & $\checkmark$ & & & \\
\hline navigation_overview_visit & & & & & $\checkmark$ & $\checkmark$ \\
\hline navigation_overview_stay & & & & & & \\
\hline sequence_content/selfass & & & $\checkmark$ & $\checkmark$ & & $\checkmark$ \\
\hline sequence_content/example & & & & & $\checkmark$ & \\
\hline sequence_content/exercise & & & & & $\checkmark$ & $\checkmark$ \\
\hline sequence_exercies/selfass & & & $\checkmark$ & $\checkmark$ & & \\
\hline sequence_exercise/example & $\checkmark$ & $\checkmark$ & & & & \\
\hline sequence_example_first & & & $\checkmark$ & $\checkmark$ & & \\
\hline sequence_assignment_first & & & $\checkmark$ & $\checkmark$ & & \\
\hline sequence_content_last & & & & & $\checkmark$ & $\checkmark$ \\
\hline sequence_example_last & & & $\checkmark$ & $\checkmark$ & & \\
\hline sequence_selfass_last & $\checkmark$ & & & & & \\
\hline sequence_exercise_last & & & & $\checkmark$ & & $\checkmark$ \\
\hline sequence_assignment_last & $\checkmark$ & $\checkmark$ & & & $\checkmark$ & $\checkmark$ \\
\hline
\end{tabular}

Another requirement for providing adaptivity is to know the learning styles of the learners. While the most often used approach in adaptive systems is to let students fill out a questionnaire which aims at detecting their learning styles, recent research is done on developing an automatic approach which identifies the learning styles based on the behaviour of students during an online course. Such an automatic student modelling approach can be more accurate by avoiding answers that are not in agreement with students' actual behaviour or preferences, given intentionally or non- 
intentionally. Moreover, students just need to use the learning system and do not have any additional effort.

The second part of this study aims at finding correlations between the behaviour of students in an online course and their learning styles. Such a correlation allows, on one hand, inferences from the learning styles to the behaviour of students. On the other hand, it allows drawing conclusions from the behaviour of the students to their learning styles. This information can be used to investigate and develop an automatic approach for detecting learning styles based on the behaviour of students in LMS.

In the following section, we introduce related work on automatic approaches for identifying learning styles.

\section{Related Work}

Some recent work already exist dealing with systems that analyse the behaviour of students in an online course and use this information to generate or update the student model with respect to learning styles.

For example, García et al. [18] proposed an automatic approach for the system SAVER. The approach considers the active/reflective, sensing/intuitive, and the sequential/global dimensions of the Felder-Silverman learning style model. The visual/verbal dimension is not incorporated since no relevant learning material is presented in the course. Overall, 11 patterns are included for the three dimensions. These 11 patterns are derived from literature. Based on the data from these patterns, Bayesian networks [19] are used to calculate the preferences of the learning style dimensions for each learner. An evaluation of the approach with 10 users shows a high degree of precision when comparing the calculated learning styles of the users with their results from the ILS questionnaire.

Another approach for automatic detection of learning styles was investigated by Cha et al. [20]. Again, they observed the behaviour of learners during an online course in an intelligent learning environment based on specific patterns. An experiment with 70 learners was conducted in order to test the effectiveness of Decision Trees [21] and Hidden Markov Models [22] for detecting learning styles according to FSLSM. For both techniques, several patterns were incorporated for each learning style dimension. Results of the experiment show a high degree of precision for two dimensions, and moderate degree of precision for the other two dimensions.

All these approaches used patterns that were derived from literature. However, most learning style models and also FSLSM are based on tradi- 
Analysing the Behaviour of Students in Learning Management Systems with Respect to Learning Styles 21

tional learning rather than on online learning. In our investigations, we look whether students with different learning styles really behave differently in learning management systems and whether a correlation exists between their behaviour and their learning styles. This information can be used to improve the selection of patterns from literature and therefore enhance the detection process of learning styles.

Furthermore, the above described approaches are developed for specific systems and therefore used only those features and patterns which are incorporated in the system. When investigating the behaviour of learners in LMS and aiming at inferring learning styles from this behaviour, we have to consider that authors have a lot of options to create courses by using or not using particular features. Therefore, it is important to have many different patterns from which information can be gained. Moreover, neither the LMS itself nor the structure of most courses is developed in consideration of learning styles. Thus, investigations about the actual behaviour of students in LMS are even more important for developing an approach that automatically detects students' learning styles.

\section{Conclusion and Future Work}

In this chapter, we presented investigations about the behaviour of students in an online course within a learning management system with respect to the students' learning styles. By analysing the behaviour of students based on predefined patterns, we found significant results for several patterns, indicating that students with different learning style preferences also behave differently in the online course. These results can act as recommendation when aiming at providing courses in LMS that fit to the learning styles of students.

Furthermore, the behaviour of students and their learning style preferences were analyzed with regard to correlations. Again, several significant correlations were found. These results additionally allow drawing conclusions from the behaviour of students to their learning styles and can be used to develop an automatic approach to detect learning style in learning management systems.

The results of our study provide information to facilitate the consideration of learning styles in learning management systems. Future work will deal on one hand with using the gathered information as basis for providing adaptivity in LMS. On the other hand, the results regarding correlations between learning styles and behaviour will be used to investigate and 
develop an approach for automatic identification of learning styles from the behaviour of students in LMS.

\section{Acknowledgements}

This research has been partly funded by the Austrian Federal Ministry for Education, Science, and Culture, and the European Social Fund (ESF) under grant 31.963/46-VII/9/2002.

\section{References}

1. Jonassen DH, Grabowski BL (1993) Handbook of individual differences, learning, and instruction. Lawrence Erlbaum Associates, Hillsdale, New Jersey

2. Felder RM, Silverman LK (1988) Learning and teaching styles in engineering education. Engineering Education 78(7):674-681. Preceded by a preface in 2002: http://www.ncsu.edu/felderpublic/ Papers/LS-1988.pdf.

3. Felder RM, Soloman BA. (1997). Index of Learning Styles questionnaire. Retrieved 30 April, 2007, from http://www.engr.ncsu.edu/learningstyles/ ilsweb.html

4. Bajraktarevic N, Hall W, Fullick P (2003) Incorporating learning styles in hypermedia environment: Empirical evaluation. In: Proceedings of the Workshop on Adaptive Hypermedia and Adaptive Web-Based Systems, Nottingham, UK, pp 41-52

5. Carver CA, Howard RA, Lane WD (1999) Addressing different learning styles through course hypermedia. IEEE Transactions on Education 42(1):3338

6. Shang Y, Shi H, Chen S-S (2001) An intelligent distributed environment for active learning. ACM Journal of Educational Resources in Computing 1(2):117

7. Papanikolaou KA, Grigoriadou M (2003) An instructional framework supporting personalized learning on the web. In: Proceedings of the International Conference on Advanced Learning Technologies. IEEE Computer Society, Los Alamitos, CA, pp 120-124

8. Paredes P, Rodríguez P (2004) A mixed approach to modelling learning styles in adaptive educational hypermedia. Advanced Technology for Learning 1(4):210-215

9. Brusilovsky P (2004) Knowledge Tree: A distributed architecture for adaptive e-learning. In: Proceedings of the International Conference on World Wide Web. ACM Press, New York, USA, pp 104-113

10. Moodle. (2007). Retrieved 30 April, 2007, from http://www.moodle.org 
Analysing the Behaviour of Students in Learning Management Systems with Respect to Learning Styles 23

11. Blackboard. (2007). Retrieved 30 April, 2007, from http://www.blackboard.com

12. WebCT. (2007). Retrieved 30 April, 2007, from http://www.webct.com/

13. Graf S, List B (2005) An evaluation of open source e-learning platforms stressing adaptation issues. In: Proceedings of the 5th International Conference on Advanced Learning Technologies. IEEE Press, pp 163-165

14. Kolb DA (1984) Experiential learning: Experience as the source of learning and development. Prentice-Hall, Englewood Cliffs, New Jersey

15. Honey P, Mumford A (1982) The manual of learning styles. Peter Honey, Maidenhead

16. Kuljis J, Liu F (2005) A comparison of learning style theories on the suitability for elearning. In: Hamza MH (ed), Proceedings of the IASTED Conference on Web Technologies, Applications, and Services. ACTA Press, pp 191-197

17. Felder RM, Spurlin J (2005) Applications, reliability and validity of the Index of Learning Styles. International Journal on Engineering Education 21(1):103112

18. García P, Amandi A, Schiaffino S, Campo M (in press) Evaluating Bayesian networks' precision for detecting students' learning styles. Computers \& Education

19. Jensen FV (1996) An introduction to Bayesian networks. Springer, New York

20. Cha HJ, Kim YS, Park SH, Yoon TB, Jung YM, Lee J-H (2006) Learning style diagnosis based on user interface behavior for the customization of learning interfaces in an intelligent tutoring system. In: Ikeda M, Ashley KD, Chan T-W (eds) Proceedings of the 8th International Conference on Intelligent Tutoring Systems, LNCS, Vol. 4053. Springer, pp 513-524

21. Dunham MH (2002) Data mining: Introductory and advanced topics. Prentice Hall, Upper Saddle River, NJ, USA

22. Rabiner LR (1989) A tutorial on hidden Markov models and selected applications in speech recognition. Proceedings of the IEEE 77(2):257-286 